GANIT J. Bangladesh Math. Soc. (ISSN 1606-3694) 37 (2017) 39-50

\title{
MATHEMATICAL ANALYSIS OF EPIDEMIOLOGICAL MODEL OF INFLUENZA A (H1N1) VIRUS TRANSMISSION DYNAMICS IN BANGLADESH PERSPECTIVE
}

\author{
Rafiqul Islam ${ }^{1, *}$, Md. Haider Ali Biswas ${ }^{2}$ and A. R. M. Jalal Uddin Jamali ${ }^{3}$ \\ ${ }^{1,3}$ Department of Mathematics, Khulna University of Engineering \& Technology, \\ Khulna-9203, Bangladesh. \\ ${ }^{2}$ Mathematics Discipline, Khulna University, Khulna-9208, Bangladesh. \\ ${ }^{*}$ Corresponding author: rafiqulislamkuet@gmail.com
}

Received 24.07.2016 Accepted 30.07.2017

\begin{abstract}
This study deals with transmission dynamics of novel influenza A (H1N1) virus to understand the evolution of its epidemic in Bangladesh. For this purpose an SEIR model has been employed to study the dynamics of A (H1N1) virus relating to data of Bangladesh. To find threshold conditions, the equilibria and stability of the equilibria of the model have been determined and also analyzed. Basic Reproductive Number $\left(\mathrm{R}_{0}\right)$ is determined relating to data of Bangladesh by which Herd Immunity Threshold has been estimated. Our numerical result suggests that vaccinating 12.69\% population of Bangladesh can control spread of the pandemic novel A (H1N1) virus when outbreak occurs.
\end{abstract}

Keywords: Basic Reproduction Number, SEIR Model, Herd Immunity Threshold, Stability, Next Generation Matrix.

\section{Introduction}

Influenza A (H1N1) virus is highly contagious and pathogenic fatal disease. A novel virus named influenza A (H1N1) virus was identified in Mexico and USA on April 2009 and the world health organization (WHO) declared it as a pandemic on June 11, 2009 [17 and 19]. In Bangladesh first confirmed case of pandemic influenza A (H1N1) virus was recorded on June 18, 2009 in a traveler who had returned from USA and 23 more cases were identified within four weeks [12]. Following the WHO declaration of pandemic level 6 with this novel strain, the Ministry of Health and Family Welfare (MOHFW) of Bangladesh launched a case-based surveillance for influenza A (H1N1) virus infection. In addition to the existing sentinel surveillance system for seasonal influenza, MOHFW imposed screening on travelers at all the ports of entry from 28 April 2009 [12]. For a new infectious disease, working out epidemiological parameters can help in decision making. A key parameter for a novel infectious disease is the Basic Reproduction Number $\left(\mathrm{R}_{0}\right)$, defined as the average number of secondary cases generated by single primary case during its entire period of infectiousness in a completely susceptible population [3]. $\mathrm{R}_{0}$ directly determines the growth rate of an epidemic and the final number of infected people and it is a predominant factor to be considered in optimal policy making. Stability of epidemiological model can be analyzed with the help of the 
value $R_{0}$ [14]. The estimation of $R_{0}$ is problematic and has gained much attention. There are few methods available to estimate $\mathrm{R}_{0}$. For determination of $\mathrm{R}_{0}$, one of the best approaches is the Next Generation Matrix method [4 and 8]. This method is provided from the well-known SEIR compartmental model.

People infected with A (H1N1) passes through an incubation period where they are not infectious and do not show any symptoms. The period of incubation for A (H1N1) virus is 1 to 4 days and the infectious period case is defined as 1 day prior to the onset of symptoms to 7 days after onset [7].The symptoms of influenza A (H1N1) virus are cough, nausea, diarrhoea, fever, chills, headache, sore throat, muscle aches, runny nose, shortness of breath, joint pain etc. [5]. The novel A (H1N1) virus poses public health and developmental challenges similar to challenges posed by communicable and chronic diseases. This has made the decision makers to face considerable uncertainties. Although vaccines are available for many infectious diseases these diseases still cause great suffering and mortality in Bangladesh. In this backdrop, further research is indispensable to ascertain the control of the wide spread of the pandemic influenza A (H1N1) virus.

The first mathematical model that can be used to describe an influenza epidemic was developed early in the 20th century by Kermack and McKendrick [15] and is known as SusceptibleInfectious-Recovered (SIR) model. Later Anderson and May [1] added a fourth compartment (latency stage) to SIR model. This extension is called the SEIR model [1]. Several authors considered this well-known SEIR or its extensions model to describe the flow (transition) of people through different compartments which represent the stages of disease, in the entire population over time [2].

In this study, this well-known SEIR model is considered to find the flow (transition) of people through different compartments regarding influenza A $(\mathrm{H} 1 \mathrm{~N} 1)$ virus in perspective of Bangladesh. By using next generation matrix method, the Basic Reproduction Number $\left(\mathrm{R}_{0}\right)$ for pandemic influenza $A(H 1 N 1)$ virus is determined regarding the data of pandemic influenza $A(H 1 N 1)$ virus of Bangladesh [12, 13 and 11]. By the help of this $\mathrm{R}_{0}$, the decision making parameter Herd Immunity Threshold $\left(\mathrm{H}_{\mathrm{T}}\right)$ is determined in perspective of Bangladesh. Moreover the disease free and endemic equilibrium of SEIR model is studied extensively according to the data considered. The system of Ordinary Differential Equations (ODEs) of SEIR model is solved numerically regarding data of Bangladesh and others simulated data. According to the numerical solutions, the trends of various aspects of the susceptible, exposed, infectious and recovered of the population regarding Bangladesh are discussed.

\subsection{Mathematical Model}

In analyzing the spread and control of infectious diseases, mathematical models have become important tools. For studying the transmission dynamics of the pandemic novel influenza A $(\mathrm{H} 1 \mathrm{~N} 1)$ virus, the model considered here is the Susceptible $(\mathrm{S}(\mathrm{t})$ )-Exposed $(\mathrm{E}(\mathrm{t})$ )-Infectious (I $(\mathrm{t})$ )-Recovered $(\mathrm{R}(\mathrm{t})$ ) compartment model or in brief SEIR model [1], Figure (1). 


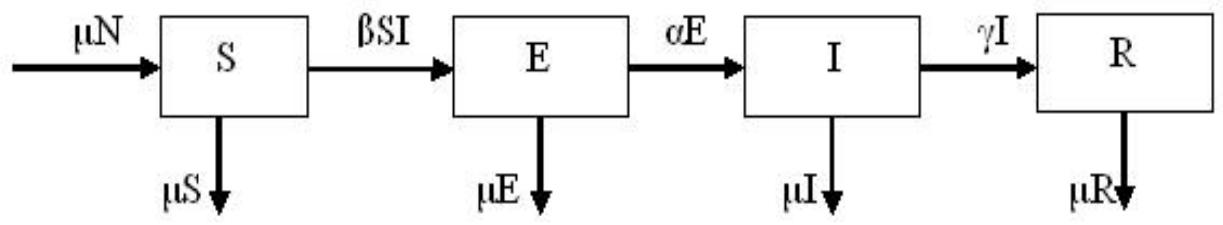

Figure 1: Flow chart of the transmission of SEIR epidemic model.

The Susceptible $(\mathrm{S}(\mathrm{t}))$ population contains the individuals who are at risk of becoming infected by influenza A (H1N1) virus. The Exposed $(\mathrm{E}(\mathrm{t}))$ class is individuals who have been infected by influenza A (H1N1) virus but yet not infectious (which show no symptoms and are not able to infect others). The Infective (I (t)) class is individuals who have been infected by influenza A $(\mathrm{H} 1 \mathrm{~N} 1)$ virus and able to transmit into the susceptible individuals. Finally Removed $(\mathrm{R}(\mathrm{t})$ ) class is individuals who have been recovered or removed from A (H1N1) virus infection [18]. For comprehensive analysis of the model, we assume birth rates and death rates occur at equal rates and the all new born are susceptible (no inherited immunity). We use symbol $\mu$ to represent average death rates as well as average birth rates (as equal). Therefore individuals are born into the susceptible class with rate $\mu \mathrm{N}$ ( $\mathrm{N}$ denotes total number of population). It is assumed that the population mix homogeneously with no restriction of age, mobility or other social factors. It is known that influenza A (H1N1) virus infected individuals will be in exposed- stage to the environment before becoming infectious. The rate at which susceptible enters into the exposed class without been infectious is $\beta S I$ (where $\beta$ be the transmission coefficient) and the rate at which exposed becomes infected is $\alpha E$ (where $\alpha$ be the exposed coefficient). The rate at which infected individuals may recover and will remain until death is $\gamma \mathrm{I}$ (where $\gamma$ be the recovery coefficient). The following system of Ordinary Differential Equations (ODEs) is used to represent this SEIR model.

$$
\left.\begin{array}{l}
\frac{d \mathrm{~S}}{d t}=\mu \mathrm{N}-\mu \mathrm{S}-\beta \mathrm{IS} \\
\frac{d \mathrm{E}}{d t}=\beta \mathrm{IS}-(\mu+\alpha) \mathrm{E} \\
\frac{d \mathrm{I}}{d t}=\alpha \mathrm{E}-(\mu+\gamma) \mathrm{I} \\
\frac{d \mathrm{R}}{d t}=\gamma \mathrm{I}-\mu \mathrm{R}
\end{array}\right\}
$$

Which satisfy the following ODE

$$
\frac{d \mathrm{~S}}{d t}+\frac{d \mathrm{E}}{d t}+\frac{d \mathrm{I}}{d t}+\frac{d \mathrm{R}}{d t}=0
$$

where $\beta>0, \alpha>0, \gamma>0 \mu>0, \mathrm{~S}+\mathrm{E}+\mathrm{I}+\mathrm{R}=\mathrm{N}$ and $t>0$ be the time . 


\subsection{Equilibrium State}

We know that Disease Free Equilibrium (DFE) or Endemic Equilibrium (EE) occurs in population according to the value of $\mathrm{R}_{0}$. Independently, one can show this in the form of biological meaningful initial conditions:

If ( $(0), E(0), I(0), R(0)) \in\left\{(S, E, I, R) \in[0, N]^{4}, S \geq 0, E \geq 0, I \geq 0, R \geq 0, S+E+I+R=N\right\}$

then $\mathrm{R}_{0} \leq 1$ implies

$$
\operatorname{Lim}_{t \rightarrow \infty}(\mathrm{S}(\mathrm{t}), \mathrm{E}(\mathrm{t}), \mathrm{I}(\mathrm{t}), \mathrm{R}(\mathrm{t}))=\text { DFE. }
$$

And $\mathrm{R}_{0}>1, \mathrm{I}(0)>0$ implies

$$
\operatorname{Lim}_{t \rightarrow \infty}(S(t), E(t), I(t), R(t))=E E .
$$

Now set $\mathrm{S}, \mathrm{E}, \mathrm{I}, \mathrm{R}$ as a proportion of the population (N), i.e.

$s(t)=\frac{S(t)}{N}, e(t)=\frac{E(t)}{N}, i(t)=\frac{I(t)}{N}, r(t)=\frac{R(t)}{N}$ and $r(t)=1-s(t)-e(t)-i(t)$, then above four ODEs (Equation. (1)) is reduced to three ODEs as follows:

$$
\left.\begin{array}{l}
\frac{\mathrm{ds}}{\mathrm{dt}}=\mu-(\mu+\beta \mathrm{i}) \mathrm{s} \\
\frac{\mathrm{de}}{\mathrm{dt}}=\beta \mathrm{si}-(\mu+\alpha) \mathrm{e} \\
\frac{\mathrm{di}}{\mathrm{dt}}=\alpha \mathrm{e}-(\gamma+\mu) \mathrm{i}
\end{array}\right\}
$$

It is known that for the steady state Equilibrium

$$
\frac{\mathrm{dK}}{\mathrm{dt}}=0, \exists \mathrm{K} \in\{\mathrm{s}, \mathrm{e}, \mathrm{i}\} \text {. }
$$

Then the above ODEs become as follows:

$$
\begin{aligned}
& \mu-(\mu+\beta i) s=0 \\
& \beta s i-(\mu+\alpha) e=0 \\
& \alpha e-(\gamma+\mu) i=0
\end{aligned}
$$

If $i=0$, then from (4) we get $s=1$ and from equation (5), we get $e=0$. Hence the Disease Free Equilibrium is as follows:

$$
(\mathrm{s}, \mathrm{e}, \mathrm{i})=(1,0,0) \text {. }
$$

Now we have to find the Endemic equilibrium. From equation (6) we have 


$$
\mathrm{e}=\frac{\gamma+\mu}{\alpha} \mathrm{i}
$$

Substituting the value of $e$ in equation (5) we get,

$$
\mathrm{s}=\frac{(\mu+\alpha)(\gamma+\mu)}{\beta \alpha}
$$

Putting the value of $s$ in equation (4) we get,

$$
\mathrm{i}=\frac{\mu \alpha}{(\mu+\alpha)(\gamma+\mu)}-\frac{\mu}{\alpha} .
$$

Therefore Endemic equilibrium is as follows:

$$
\left(\mathrm{s}^{*}, \mathrm{e}^{*}, \mathrm{i}^{*}\right)=\left\{\frac{(\mu+\alpha)(\gamma+\mu)}{\beta \alpha}, \frac{(\gamma+\mu)}{\alpha}\left(\frac{\mu \alpha}{(\mu+\alpha)(\gamma+\mu)}-\frac{\mu}{\alpha}\right), \frac{\mu \alpha}{(\mu+\alpha)(\gamma+\mu)}-\frac{\mu}{\alpha}\right\}
$$

\subsection{Parameter Estimation for Influenza A (H1N1) Virus}

It is stated earlier that the Basic Reproduction Number $\mathrm{R}_{0}$, for our compartmental SEIR model, is estimated by Next Generation Matrix method. In the Next Generation Matrix (Operator) method, $\mathrm{R}_{0}$ is defined as the spectral radius of Next Generation Matrix (Operator) $\mathbf{F V}^{-1}$ where $\mathbf{F}$ be the matrix formed by new infection individuals in the compartments and $\mathbf{V}$ be the matrix formed by the net infected individuals in the compartments. In our SEIR model, the numbers of infected compartments are two namely Exposed compartment and Infected compartment. So from the SEIR model (as we have two types of compartments - infected and non-infected), we get

$\mathrm{F}_{1}(\overline{\mathrm{x}})=\beta \mathrm{IS}, \mathrm{F}_{2}(\overline{\mathrm{x}})=0, \quad \mathrm{~V}_{1}(\overline{\mathrm{x}})=(\mu+\alpha) \mathrm{E}, \mathrm{V}_{2}(\overline{\mathrm{x}})=(\mu+\alpha) \mathrm{I}-\alpha \mathrm{E}$

Hence $\mathrm{F}=\left(\frac{\partial \mathrm{F}_{\mathrm{i}}\left(\mathrm{x}_{0}\right)}{\partial \mathrm{x}_{\mathrm{j}}}\right)=\left[\begin{array}{ll}0 & \beta \\ 0 & 0\end{array}\right]$ and $\mathbf{V}=\left(\frac{\partial \mathrm{V}_{\mathrm{i}}\left(\mathrm{x}_{0}\right)}{\partial \mathrm{x}_{\mathrm{j}}}\right)=\left[\begin{array}{cc}(\mu+\alpha) & 0 \\ -\alpha & (\mu+\gamma)\end{array}\right]$

Then $\mathbf{F V}^{-1}=\left[\begin{array}{cc}\frac{\beta \alpha}{(\mu+\gamma)(\mu+\alpha)} & \frac{\beta}{\mu+\gamma} \\ 0 & 0\end{array}\right]$

Therefore the spectral radius of $\mathbf{F V}^{-1}$ is $\frac{\beta \alpha}{(\mu+\gamma)(\mu+\alpha)}$. Hence the Basic Reproduction Number is as follows:

$$
\mathrm{R}_{0}=\frac{\beta \alpha}{(\mu+\gamma)(\mu+\alpha)}
$$

It is known that the expected duration of infectious is the inverse of the removal rate [9 and 20]. According to the definition [see 9 and 10], the removal rate $(\gamma)$, Exposed rate $(\alpha)$ and transmission rate $(\beta)$ are given as follows: 


$$
\begin{aligned}
& \gamma=\frac{1}{\text { mean infectious period }} \\
& \alpha=\frac{1}{\text { mean latancy period }} \\
& \beta=\frac{\text { effective contact }}{\text { total contact }}
\end{aligned}
$$

\subsection{Stability Analysis}

The local stability of the DFE and EE steady states are calculated by simplifying the system of ODEs (3). From the system of ODEs (3), we have the Jacobian matrix as follow:

$$
J=\left[\begin{array}{ccc}
-(\mu+\beta i) & 0 & -\beta s \\
\beta i & -(\mu+\alpha) & \beta s \\
0 & \alpha & -(\mu+\gamma)
\end{array}\right]
$$

So the Jacobian at the Disease Free Equilibrium i.e at $(\mathrm{s}, \mathrm{e}, \mathrm{i})=(1,0,0)$ is

$$
\mathrm{J}_{\mathrm{DFE}}=\left[\begin{array}{ccc}
-\mu & 0 & -\beta \\
0 & -(\mu+\alpha) & \beta \\
0 & \alpha & -(\mu+\gamma)
\end{array}\right]
$$

Now the characteristic equation of $\mathrm{J}_{\mathrm{DFE}}$ is

$$
\left|\left(\mathrm{J}_{\mathrm{DEE}}-\lambda \mathrm{I}\right)\right| \lambda^{3}+b_{1} \lambda^{2}+b_{2} \lambda+b_{3}
$$

where $b_{1}=(3 \mu+\gamma+\alpha)$

$$
\begin{aligned}
& \left.\mathrm{b}_{2}=[(\mu+\gamma)(\mu+\alpha)-\beta \alpha+\mu(2 \mu+\gamma+\alpha))\right] \\
& \mathrm{b}_{3}=\mu[(\mu+\gamma)(\mu+\alpha)-\beta \alpha]
\end{aligned}
$$

If $b_{1}>0, b_{3}>0$ and $b_{1} b_{2}-b_{3}>0$ are true, then by Routh-Hurwitz criterion, all the roots of the characteristic equation have negative real part which means stable equilibrium [16]. Using $R_{0}$ (from equation (8)) in $b_{2}$ and $b_{3}$, we get $b_{2}=\beta \alpha\left(\frac{1}{R_{0}}-1\right)+\mu(2 \mu+\gamma+\alpha)$ and $b_{3}=\mu \beta \alpha\left(\frac{1}{R_{0}}-1\right)$.

Therefore

$$
\mathrm{b}_{1} \mathrm{~b}_{2}-\mathrm{b}_{3}=\left(\frac{1}{\mathrm{R}_{0}}-1\right)(2 \mu)\left(+\alpha \beta \gamma+\beta \alpha^{2}\right\}+\mu(2 \mu+\gamma+\alpha)(3 \mu+\gamma+\alpha) .
$$

Here $b_{1}>0$ is always true. Now $b_{3}>0$ and $b_{1} b_{2}-b_{3}>0$ will be true only iff $R_{0}<1$. So Disease Free Equilibrium is stable iff $\mathrm{R}_{0}<1$ otherwise it is unstable.

By the help of equation (8) we rewrite the Endemic Equilibrium equation (7) as follow: 


$$
\left(\mathrm{s}^{*}, \mathrm{e}^{*}, \mathrm{i}^{*}\right)=\left[\frac{1}{\mathrm{R}_{0}}, \frac{\mu\left(\mathrm{R}_{0}-1\right)}{\mathrm{R}_{0}(\mu+\alpha)}, \frac{\mu\left(\mathrm{R}_{0}-1\right)}{\beta}\right]
$$

By using the value of $\left(\mathrm{s}^{*}, \mathrm{e}^{*}, \mathrm{i}^{*}\right)$ in equation (12), we have the Jacobian at endemic equilibrium point as follow:

$$
\mathrm{J}_{\mathrm{EE}}=\left[\begin{array}{ccc}
-\mu \mathrm{R}_{0} & 0 & \frac{-(\mu+\gamma)(\mu+\alpha)}{\alpha} \\
\mu\left(\mathrm{R}_{0}-1\right) & -(\mu+\alpha) & \frac{-(\mu+\gamma)(\mu+\alpha)}{\alpha} \\
0 & \alpha & -(\mu+\gamma)
\end{array}\right]
$$

Therefore the characteristic equation of $J_{E E}$ is

$$
\left|\left(\mathrm{J}_{\mathrm{EE}}-\lambda \mathrm{I}\right)\right|=\lambda^{3}+\mathrm{p}_{1} \lambda^{2}+\mathrm{p}_{2} \lambda+\mathrm{p}_{3},
$$

where $\mathrm{p}_{1}=\alpha+\gamma+\left(2+\mathrm{R}_{0}\right) \mu$

$$
\begin{aligned}
& \mathrm{p}_{2}=\mathrm{R}_{0} \mu(2 \mu+\alpha+\gamma) \\
& \mathrm{p}_{3}=\mu\left(\mathrm{R}_{0}-1\right)\left[\mu^{2}+\mu(\alpha+\gamma)+\alpha \gamma\right]
\end{aligned}
$$

Again if the coefficients of characteristic equation $p_{1}>0, p_{3}>0$ and $p_{1} p_{2}-p_{3}>0$ are true, then by Routh-Hurwitz criterion, all the roots of the characteristic equation have negative real parts which means a stable equilibrium [8]. It is notified that if $R_{0}>1$ then both $\mathrm{p}_{1}$ and $\mathrm{p}_{3}$ become positive quantities. Now for the third condition $\mathrm{p}_{1} \mathrm{p}_{2}-\mathrm{p}_{3}>0$, we have

$$
\mathrm{p}_{1} \mathrm{p}_{2}-\mathrm{p}_{3}=\mu\left[\mathrm{R}_{0}\left\{(3 \mu+\alpha+\gamma)(-\alpha+\gamma)+\mu^{2}\left(3+2 \mathrm{R}_{0}\right)+\gamma^{2}\right\}+\mu^{2}+\mu(\alpha+\gamma)+\alpha \gamma\right],
$$

which is greater than zero for all parameters value along with $R_{0}>1$. Therefore by RouthHurwitz criterion when $\mathrm{R}_{0}>1$ the endemic steady state is stable.

\subsection{Herd Immunity Threshold}

To control transmission of the disease, the population has to be immunized. The percentage of the population that needs to be immunized for controlling the transmission is the Herd Immunity Threshold (HIT) [6]. We have proposed the HIT $\left(\mathrm{H}_{\mathrm{T}}\right)$ as the sole immunization strategy. It protects directly the immunized individuals from infection and also provides protection of being susceptible individuals. To evaluate HIT, we use the equation, given in [3], as follows:

$$
H_{T}=1-\frac{1}{R_{0}}
$$

\subsection{Model Analysis}

In order to evaluate the Herd Immunity Threshold as well as numerical analysis of the SEIR models, we have considered the information about influenza A (H1N1) virus as defined in [7] and real numerical data in perspective of Bangladesh [12, 13 and 11]. The latency period of A (H1N1) 
virus is 1 to 4 days and infectious period is one day prior to on set of symptoms to 7 days after symptom onset [7]. So the mean latency period of A (H1N1) virus is 2 days and mean infectious period is 3.5 days. Therefore by using equations (9) and (10), we have $\gamma=0.2857, \alpha=0.5$. According to [12], from 18 June 2009 to 20 July 2009, 84 individuals had been tested and among them 24 individuals were found influenza A (H1N1) virus positive. Again for month July 2015, 258 individuals have been tested and among them 100 individuals were found influenza A (H1N1) virus positive [13]. Therefore, we have

$$
\beta_{1}=\frac{24}{84}, \beta_{2}=\frac{100}{258} \text { and } \beta=\frac{\beta_{1}+\beta_{2}}{2}=0.3374 \text {. }
$$

We have the death rate of population of Bangladesh is $\mu=0.00564$ and the total population of Bangladesh is $N=166280712$ [11]. Using the value of $\beta, \alpha, \gamma$ and $\mu$ in (8) we get $R_{0}=1.1453$. So Disease Free Equilibrium $(\mathrm{s}, \mathrm{e}, \mathrm{i})=(1,0,0)$ is unstable as $\mathrm{R}_{0}(=1.1453)>1$. By substituting the above value in equation (13), we have Endemic Equilibrium

$$
\left(\mathrm{s}^{*}, \mathrm{e}^{*}, \mathrm{i}^{*}\right)=\left[\frac{1}{\mathrm{R}_{0}}, \frac{\mu\left(\mathrm{R}_{0}-1\right)}{\mathrm{R}_{0}(\mu+\alpha)}, \frac{\mu\left(\mathrm{R}_{0}-1\right)}{\beta}\right]=(0.8731,0.001415,0.002428)
$$

which is stable as $R_{0}(=1.1453)>1$. Moreover by Equation (14), the Herd Immunity Threshold is calculated as follows:

$$
H_{T}=1-\frac{1}{\mathrm{R}_{0}}=0.1269 .
$$

Now the sensitivity will be analyzed for both Disease Free Equilibrium and Endemic Equilibrium points of the SIER model in perspective of Bangladesh. For these experiments the death rate as well as the expose rate is considered to be fixed. On the other hand the transmission rate as well as the recovery rate is changed as shown in the Tables I. At first, the value of recovery rate $(0.2857)$ is kept unchanged whereas the corresponding values of transmission rate are changed. Secondly keeping the value of transmission rate (0.3374) unchanged, we have varied the values of recovery rate. Finally we have calculated the $R_{0}$ values for each changed values of $(\gamma)$. The experimental results are displayed in the Table I. It is observed in the Table I that when the transmission rate $\left({ }_{R}\right)$ decreases $(0.3374 \rightarrow 0.25)$ with unchanged values of other parameters, then the Disease Free Equilibrium approaches unstable to stable and Endemic Equilibrium becomes stable from unstable as the value of $\mathrm{R}_{0}$ decrease from 1.1453 to less than one. Again keeping unchanged the values of all parameters except the value of recovery rate, we have observed that for the increasing of the value of recovery rate $(0.2857 \rightarrow 0.5)$, the Disease Free Equilibrium again approaches from unstable to stable as well as Endemic Equilibrium becomes unstable from stable as the value of $\mathrm{R}_{0}$ decreases to less than one. That is if transmission rate will decrease or recovery rate will increase then the Disease Free Equilibrium becomes stable as well as Endemic Equilibrium approaches to unstable in perspective of Bangladesh. This implies that the disease will not spread out. What happen if we will consider the reverse strategy? From the experimental results, given in 
the Table I, we observe that when the transmission rate $(\beta)$ increased or recovery rate $(\gamma)$ decreased then $\mathrm{R}_{0}$ becomes greater than one and consequently the Disease Free Equilibrium is found unstable as well as Endemic Equilibrium becomes stable from unstable as well. Which implies disease will spread out.

Table I. Sensitivity analysis of the Disease Free Equilibrium state and Endemic Equilibrium state.

\begin{tabular}{|l|l|l|l|l|l|l|}
\hline$\mu$ & $\beta$ & $\alpha$ & $\gamma$ & $\mathrm{R}_{0}$ & Nature of DFE state & Nature of EE state \\
\hline 0.00564 & 0.35 & 0.5 & 0.2857 & 1.1879 & Unstable & Stable \\
\hline 0.00564 & 0.3374 & 0.5 & 0.2857 & $\mathbf{1 . 1 4 5 3}$ & Unstable & Stable \\
\hline 0.00564 & 0.30 & 0.5 & 0.2857 & 1.0824 & Unstable & Stable \\
\hline 0.00564 & $\mathbf{0 . 2 9 4 6 2 6}$ & 0.5 & 0.2857 & 1.00 & Critical value & Critical value \\
\hline 0.00564 & $\mathbf{0 . 2 7}$ & 0.5 & 0.2857 & 0.9164 & Stable & Unstable \\
\hline 0.00564 & $\mathbf{0 . 2 5}$ & 0.5 & 0.2857 & 0.8485 & Stable & Unstable \\
\hline 0.00564 & 0.3374 & 0.5 & 0.3 & 1.09156 & Unstable & Stable \\
\hline 0.00564 & 0.3374 & 0.5 & $\mathbf{0 . 3 2 7 9 9}$ & 1.00 & Critical value & Critical value \\
\hline 0.00564 & 0.3374 & 0.5 & $\mathbf{0 . 3 5}$ & 0.9238 & Stable & Unstable \\
\hline 0.00564 & 0.3374 & 0.5 & $\mathbf{0 . 5}$ & 0.1668 & Stable & Unstable \\
\hline 0.00564 & 0.3374 & 0.5 & 0.125 & 2.5539 & Unstable & Stable \\
\hline
\end{tabular}

In perspective of Bangladesh, now we will find the critical value of Transmission rate and the critical value of recovery rate by keeping constant the values of other parameters $(\mu, \alpha, \gamma)$ and $(\mu$, $\alpha, \beta)$ respectively. To find the critical value of Transmission rate $\left(\beta^{*}\right)$ we set $\mathrm{R}_{0}=1$ (by keeping fix other parameters), in equation (8) we have $\beta^{*}=0.294626$. Now to find the critical value of Recovery rate $\left(\gamma^{*}\right)$, we again set $\mathrm{R}_{0}=1$ (by keeping fix other parameters), in equation (8) we have $\gamma^{*}=0.32799$. The experimental results regarding $\beta^{*}$ and $\gamma^{*}$ are also displayed in the Table I. Therefore, if all the parameters are unchanged except the transmission rate, the disease will be spread out or controlled according to the values of transmission rate is greater or less than the critical value of $\beta^{*}=0.294626$. Similarly, if all the parameters are unchanged but only the values of recovery rate are varied, then disease is spread out or controlled according to the values of recovery rate is less or greater than the critical value of $\gamma^{*}=0.32799$.

Here $\mathrm{R}_{0}>1$ revealed that the state of disease free equilibrium is unstable and the state of endemic equilibrium is stable. In the case of unstable state of disease free equilibrium as well as in the case of stable state of endemic equilibrium, diseases spread out. To control the spread out of the disease, we need to calculate Herd Immunity Threshold. In perspective of Bangladesh, we have calculated the Herd Immunity Threshold, which is 0.1269 . 

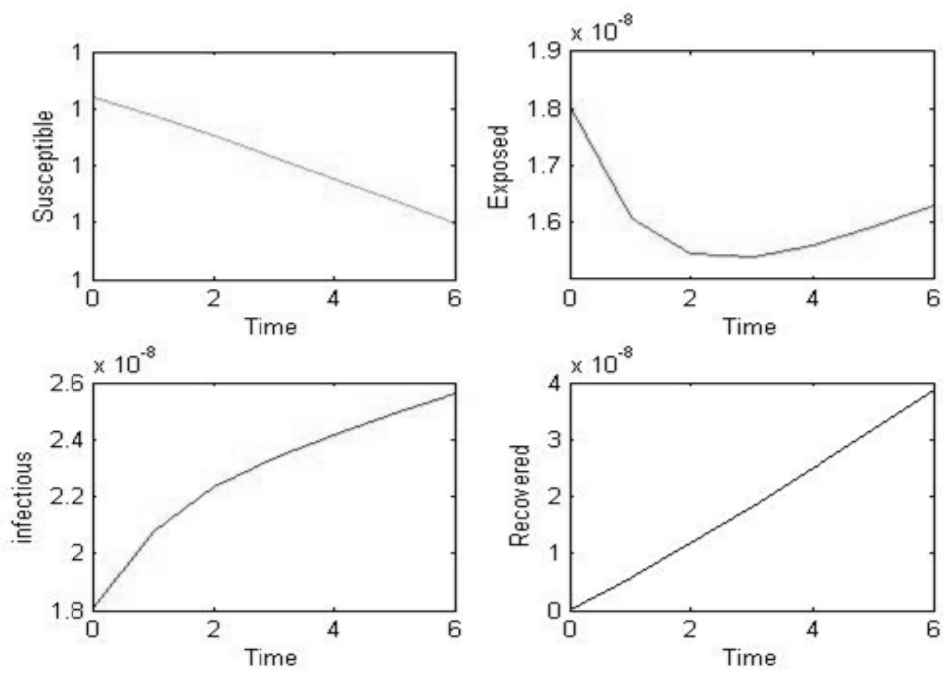

Figure 2: Dynamics of the various compartments at the initial outbreak of A (H1N1).

Moreover, we have performed numerical simulation of the model. For the numerical simulation of the SIER model (the system of ODEs (3)), in perspective of Bangladesh on March 2014, we have, $S(0)=166280706, E(0)=3$ (assumed), $I(0)=3$ and $R(0)=0$ [10]. So dividing each of the terms by the total population of Bangladesh, $\mathrm{N}=166280712$ [17], we have $s(0)=0.9999999639, e(0)$ $=0.00000001804, i(0)=0.00000001804$ and $\mathrm{r}(0)=0$ respectively. It is noted that regarding the data of Bangladesh the value of $\mu=0.00564, \alpha=0.5, \beta=0.3374$ and $\gamma=0.2857$ are calculated in the section 2.6. The numerical solutions of the system of ODEs (3) are shown in the Figure 2. It is observed in the Figure 2 that the initial proportion of infectious has minimal effect on susceptible. One of the cause of this insignificance effect on susceptible may be that the value of initial infectious $i(0)=0.00000001804$ is very small.

What happen if the proportion of infective is significantly large, keeping unchanged the value of the parameters? For this experiment, we have changed the proportion of initial infective to $i(0)$ $=0.4$ as well as proportion of initial susceptible $s(0)=0.5999999639$ (but $e(0)=0.00000001804$ is considered unchanged), around the endemic equilibrium point for a period of 5 and 16 months. The simulated results of 16 months period are shown in Figure 3.We observe in Figure 3 that when the population of infectious is increased to proportion 0.4, around the neighborhood of endemic equilibrium, the proportion of exposed individuals initially increased from 0 to 0.06 in two months then decreased gradually to a 0.01 by 16 months. On the other hand the proportion of susceptible declines from 0.5999999639 to a minimum value 0.31 by 16 months. The proportion of recovered also increases monotonically with time and has reached at point 0.62 by 16 months. We also observed that the recovered population is equal to infective around the 2.5 months at a value 0.24 and to susceptible around 5.9 months at a value 0.4 from the outbreak. The simulated results of 5 months period reveal similar trend with simulated results of 16 months. It is also 
observed in both periods that exposed are maximum at 2 months and after 2 months the proportion of exposed monotonically decrease.

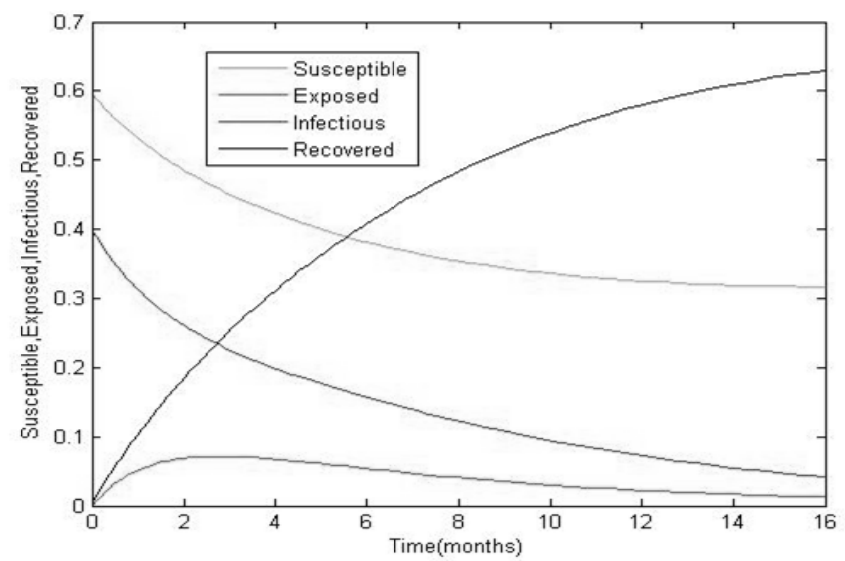

Figure 3: Numerical simulated values of various compartments within 16 months periods regarding SEIR model.

\subsection{Discussion}

The estimated Basic Reproduction Number $\left(\mathrm{R}_{0}\right)$ for the SEIR epidemiological model is greater than one in perspective of Bangladesh. This indicates that disease will spread out. The sensitivity analysis revealed that whenever the transmission rate is increased or recovery rate is decreased, the disease spread, but whenever the transmission rate is decreased or recovery rate is increased then the value of estimated $\mathrm{R}_{0}$ becomes less than one, this implies that the disease dies out. In the experiments we have also estimated the critical value of transmission rate (when other parameters except $\beta$ assumed unchanged) and recovery rate (when other parameters except $\gamma$ assumed unchanged). If it is possible to apply some mechanism (like closing school, preventing mass gathering etc) by which the value of $\beta$ can be made less than $\beta^{*}(0.294626)$ then spread out of disease can be controlled. Similarly if it is possible to apply some mechanism (like applying drug, environment support etc) by which the value of $\gamma$ can be made greater than $\gamma^{*}(\mathbf{0 . 3 2 7 9 9})$ then spread out of the disease can be controlled.

From the simulation Figure 2, it is found that the initial proportion of invectives has minimal effect on the various compartments. As the proportion of infective was increased to 0.4 (shown in Figure 3) around the neighborhood of endemic equilibrium state, the SEIR model exhibited a decline in the proportion of susceptible. This means that as more and more people infected with A (H1N1) virus, the disease will be endemic in that region. Furthermore it is observed that the recovered population increases with time monotonically. This result implies that even though the susceptible population was infected, a high amount of them recovered quickly which provide herd immunity. In this study our controlling strategy is vaccinating for which we have found the Herd Immunity Threshold. In perspective of Bangladesh, for influenza A (H1N1) virus we have found that the 
Herd Immunity Threshold is 0.1269 , by using SEIR model. This means that vaccinating $12.69 \%$ population of Bangladesh can control spreading of the pandemic novel A (H1N1) virus when outbreak occurs.

\section{REFERENCES}

[1] Anderson R. M. and May R. M., Infectious Diseases of Humans: Dynamics and Control, Oxford University Press, Oxford, 1991.

[2] Coburn B. j., Wagner B. G. and Blower S., Modeling influenza epidemics and pandemics: insights into the future of swine flu (H1N1), BMC Med, 7(30), June 2009.

[3] Dickmann O. and Heeserbeek J. A. P., Mathematical Epidemiology of Infectious Disease: Model Building Analysis and Interpretation, John Wiley, New York, 2000.

[4] Driesschea P.D. and Watmough J., Reproduction numbers and sub-threshold endemic equilibria for compartmental models of disease transmission, math Biosci, 180: 29-48, 2002.

[5] Eccles R., Understanding the symptoms of the common cold and influenza, The lancet infectious diseases Vol.5, No.11, 718-25, November 2005.

[6] Fine P., Eames K., and Heymann D. L., Herd Immunity: A Rough Guide, Clinical Infectious Diseases, 52(7): 911-916, 2011

[7] Gu Y., Komiya N., Kamiya H., Yasui Y., Taniguchi K., Okabe N., Pandemic A(H1N1) 2009 transmission during pre-symptomatic phase, Japan, Emerging infectious diseases, 17(9), September 2011.

[8] Heffernan J. M., Smith R. J. and Wahl L. M., Perspectives on the basic Reproduction ratio, Journal of Royal Society Interface, 2: 281-293, 2005.

[9] Hethcote H. W., The mathematics of Infectious Diseases, SIAM Review, 42(4): 599-653, December, 2000.

[10] http://en.wikipedia.org/wiki/Transmission_risks_and_rates/[Accessed 20.08.2015].

[11] https://en.wikipedia.org/wiki/Demographics_of_Bangladesh / [Accessed 20.08.2015].

[12] ICDDR, B., www.icddrb.org/component/content/article/10030-news/2007-h1n1-in. Bangladesh-thefirst-month.

[13] IEDCR, http://www.iedcr.org/index.php?option=com_content\&view=article\&id=130 \& Itemid=86 [Accessed 09.08.2015].

[14] Islam R., Biswas H. A. M. and Jamali A. R. M. J. U, Stability Analysis of Steady States for Epidemiological Model of Influenza A (H1N1) Virus Transmission Dynamics in Perspective of Bangladesh, 19th International Mathematics Conference, 18-20 December 2015, BRACU Campus, Dhaka, Bangladesh.

[15] Kermack W. O and McKendrick A. G, A contribution to the mathematical theory of epidemics. Proc. Roy Soc Lond 1927.

[16] Murry J. D., Mathematical Biology I. An Introduction, Third Edition, Springer-Verlag Berlin Heidelberg, 2002.

[17] Tan X., Yuan L., Zhouu J., Zeheng Y. and Yang F., Modelling the initial transmission Dynamics of influenza A H1N1 in Gungdon Province, China, International Journal of Infectious Diseases, 17: e479-e-484, 2013

[18] Uhavax (2001) History of epidemics and plagues. http://uhavax.hartford.edu/bugl/histepi.htm [Accessed: 10.08.2015].

[19] www.who.int/pmnch/media/news/2009/20090611_who/en/ [Accessed 01.09.2015].

[20] www.ibmathsresources.com/2014/05/17/modelling-infectious-diseases/ [Accessed 01.09.2015]. 